\title{
Postoperative changes of the free pericardial fat pad for bronchial stump coverage
}

\author{
Takuya Nagashima ${ }^{1,2}$, Hiroyuki Ito ${ }^{1}$, Joji Samejima ${ }^{1}$, Daiji Nemoto ${ }^{1}$, Daisuke Eriguchi ${ }^{1}$, \\ Haruhiko Nakayama ${ }^{1}$, Tetsukan Woo ${ }^{2}$, Munetaka Masuda ${ }^{2}$
}

${ }^{1}$ Department of Thoracic Surgery, Kanagawa Cancer Center, Yokohama, Japan; ${ }^{2}$ Department of Surgery, Yokohama City University, Yokohama, Japan

Contributions: (I) Conception and design: T Nagashima, T Woo, M Masuda; (II) Administrative support: T Nagashima, J Samejima, D Nemoto, D Eriguchi, H Ito, H Nakayama; (III) Provision of study materials or patients: T Nagashima, H Ito; (IV) Collection and assembly of data: T Nagashima; (V) Data analysis and interpretation: T Nagashima, T Woo; (VI) Manuscript writing: All authors; (VII) Final approval of manuscript: All authors.

Correspondence to: Takuya Nagashima. Department of Thoracic Surgery, Kanagawa Cancer Center, 2-3-2 Nakao, Asahi-ku, Yokohama City 241-8515, Japan. Email: naga16@kcch.jp.

Background: Bronchopleural fistula (BPF) remains a serious complication after surgery for lung cancer with bronchial resection. A free pericardial fat pad (FPFP) is applied in high-risk cases to reduce BPF frequency. BPF may occur 6 months after surgery. Thus, we evaluated the residual FPFP volume at 6 months after surgery to estimate the residual FPFP ratio and determine the amount of FPFP to be harvested during surgery.

Methods: We retrospectively investigated 40 patients who underwent lobectomy with bronchial stump coverage using FPFP. During surgery, the volume of the harvested FPFP was measured and the FPFP was affixed to the bronchial stump. Further, 6 months after surgery, the residual volume of the installed FPFP was analyzed using a three-dimensional volume analyzer and the residual ratio was calculated. We also evaluated clinicopathological factors influencing the resected FPFP and residual ratio.

Results: The median resected FPFP volume was 11 [3-40] mL. During multivariate analysis, body mass index and surgical approach were found to be significant factors associated with the resected FPFP volume. The median residual FPFP volume was $4.3(0.4-15.5) \mathrm{mL}$. The median residual ratio was $0.39(0.13-0.66)$. The resected FPFP volume was significantly associated with the residual volume $(\mathrm{P}<0.001)$ but not with the residual ratio $(\mathrm{P}=0.811)$. No factor was associated with the residual ratio.

Conclusions: In all cases, residual FPFP was confirmed at 6 months after surgery and the residual ratio was $40 \%$. It is necessary to determine the volume of FPFP to be harvested while carefully considering the shrinkage ratio.

Keywords: Bronchial fistula; pericardial fat; lung cancer; imaging; three-dimensional (3D)

Submitted Sep 19, 2019. Accepted for publication Nov 22, 2019.

doi: $10.21037 /$ jtd.2019.11.81

View this article at: http://dx.doi.org/10.21037/jtd.2019.11.81

\section{Introduction}

Bronchopleural fistula (BPF) remains a rare but serious complication that can appear after surgery for lung cancer with bronchial resection $(1,2)$. Such may result in fatal respiratory failure, pyothorax, or bronchopulmonary artery fistula. Some risk factors for the occurrence of a bronchial fistula have been reported, including male sex, diabetes mellitus, smoking, induction therapy, advanced-stage lung cancer, right lower lobectomy, bronchoplasty, and acute exacerbation of interstitial pneumonia $(1,3)$.

Several autologous tissue materials for covering the 
bronchial stump have been explored to reduce the risk of this complication. However, the decision about the material to be used in this context varies among physicians and institutions, attributed to the lack of concrete evidence concerning the effectiveness of certain materials and their coverage (4). Getman et al. reported that viable autologous tissue contributes to the low incidence of BPF (5); thus, the blood flow of these materials is often considered important.

Meanwhile, the effectiveness of the free pericardial fat pad (FPFP), which has no blood flow, has been examined. According to previous research, the FPFP remains in place for a certain period after lung surgery, but the occurrence of BPF under FPFP coverage has not been discussed $(6,7)$. FPFP offers easier handling even under video-assisted thoracoscopic surgery (VATS) or robotic-assisted surgery and leads to markedly less damage during harvest; thus, it is less invasive when compared with collection of the intercostal muscle or omentum. Based on this evidence, we typically incorporate FPFP in the prevention of BPF in patients with known risk factors.

Our hypothesis is that FPFP can prevent BPF in the long-term period. BPF usually develops within 1 or 2 months after surgery; however, it has also been reported to appear at 6 months after surgery (2). Therefore, FPFP must maintain its volume for 6 months or longer after surgery. To prove this hypothesis, it is necessary to show that there is a significant difference in the incidence of BPF between groups treated with or without FPFP. However, because the incidence of BPF itself is low, many trials may be required. Therefore, as an initial step, we confirmed two phenomena. One was the remaining of FPFP in the long-term period during which BPF can occur. The other was the residual ratio used to estimate the ideal volume of harvested FPFP. However, although some reports have shown that the FPFP can shrink over time but remain to some degree, we found that the number of remnant cases is typically small and the residual ratio in the long-term is unclear. The estimation of the amount of residual FPFP at 6 months after surgery would provide a reference to determine the optimal amount of FPFP to be harvested during surgery such that it would remain in place for as long as needed.

Recent developments in imaging technology have facilitated the precise quantification of intrathoracic structures (8). In this study, we measured the residual FPFP volume using a three-dimensional (3D) volume analyzer. Furthermore, we also examined clinical factors that may influence the resected FPFP and residual ratio.

\section{Methods}

\section{Patients}

We retrospectively investigated 40 patients who underwent lobectomy and radical mediastinal lymph node dissection with bronchial stump coverage using FPFP between May 2015 and October 2018 at the Kanagawa Cancer Center in Yokohama, Japan. During this period, 705 lobectomies were performed and four cases of BPF were reported. Two cases did not include any coverage, whereas one case involved covering the area with intercostal muscle, and one case used a pericardial fat pad with pedicle. The selection of covering material varied among surgeons, and no definitive criteria were established. The institutional review board of Kanagawa Cancer Center approved this retrospective study (clinical registration no. 2019EKI-50). The requirement for patient consent was waived because of the retrospective nature of the study.

All cases of BPF had at least one risk factor for the condition. In VATS, FPFP was applied only during right lower lobectomy conducted using commercial mechanical staplers, and there were no cases of bronchoplasty reported. In patients who underwent thoracotomy, FPFP was used among those with a risk of BPF, including bronchoplasty. Of note, Prolene suture 3-0 (Ethicon Inc., Somerville, NJ, USA) was used for bronchoplasty. The study participant characteristics are shown in Table 1. Only one patient received preoperative radiation. There were no R1 or R2 cases, and no cases required prolonged ventilation.

\section{Operative technique and the measurement of resected FPFP volume (Figure 1)}

In all cases, complete mediastinal lymph node dissectionthat is, systematic nodal dissection (SND) or lobe-specific nodal dissection-was performed, and there were no cases of lymph node sampling. Following lung resection or bronchoplasty, a sealing test was completed, confirming the absence of air leakage from the bronchial stump or anastomosis site. Subsequently, pericardial fat was harvested using an electric cautery or energy device without a vascular pedicle. The size of the FPFP appropriate to cover the whole bronchial stump was predicted and collected by surgeons. No additional incisions in the chest wall including in the intercostal muscle were made.

For the measurement of the resected FPFP volume, FPFP was placed in a $50-\mathrm{mL}$ syringe with $20 \mathrm{~mL}$ saline, and the volume without saline was confirmed. 
Table 1 Clinical characteristics of 40 patients with bronchial stump FPFP coverage

\begin{tabular}{|c|c|}
\hline Characteristics & Value \\
\hline Gender (male*/female) & $28 / 12$ \\
\hline BMI, median (range) & $22.4(17.1-29.1)$ \\
\hline Smoking habit (yes*/no) & $33 / 7$ \\
\hline Diabetes mellitus* & $7(17.5)$ \\
\hline Hyperlipidemia & $12(30.0)$ \\
\hline Cardiac diseases & $8(20.0)$ \\
\hline Respiratory diseases (COPD, IP, asthma) & $18(45.0)$ \\
\hline Clinical $N$ status* $(0 / 1,2)$ & $27 / 13$ \\
\hline Surgical approach (VATS/thoracotomy) & $23 / 17$ \\
\hline Tumor size (cm), median (range) & $3.0(0.8-3.7)$ \\
\hline \multicolumn{2}{|l|}{ Mode of operation } \\
\hline Right upper lobectomy/right lower lobectomy*/left lower lobectomy/bilobectomy ${ }^{\star}$ & $9 / 26 / 4 / 1$ \\
\hline Bronchoplasty*, n (\%) & $9(22.5)$ \\
\hline $\mathrm{LSD}^{\star} / \mathrm{SND}^{*}$ & $24 / 16$ \\
\hline Operation time (min), median [range] & 165 [117-303] \\
\hline
\end{tabular}

*, reported risk factors for BPF. FPFP, free pericardial fat pad; BMI, body mass index; VATS, video-assisted thoracoscopic surgery; COPD, chronic obstructive pulmonary disease; IP, interstitial pneumonia; LSD, lobe-specific nodal dissection; SND, systemic nodal dissection.

Subsequently, one stitch of Prolene suture 3-0 was threaded through the center of the bronchial stump and the center of FPFP was then penetrated with both end needles of said suture. Next, the stitch was tied and the center of the FPFP was fixed to the corresponding area of the bronchial stump. Finally, the margin of the FPFP was fixed with fibrin glue and polyglycolic acid. In all cases, a 19- or 20-French chest drain was placed in the thoracic cavity and later removed following confirmation of the absence of air leakage and a decrease in pleural effusion of less than $250 \mathrm{~mL} /$ day. We determined the condition of patients at 1,3 , and 6 months after surgery through X-ray examination at outpatient clinics.

\section{Analysis of residual volume and calculation of the residual ratio}

Six months after surgery, computed tomography (CT) was performed, and high-resolution CT images with a section thicknesses of 1 to $2 \mathrm{~mm}$ were obtained. These CT images were acquired using a 16-row or 320-row multislice CT scanner (Aquilion 16 Super Heart or Aquilion ONE CT scanner; Toshiba Medical Systems, Tochigi, Japan), with or without enhancement.

We recognized a residual FPFP around the bronchial stump or anastomosis site as a fat density nodule, considering that there should be no fat remaining in the area following complete lymph node dissection. The amount of residual FPFP volume was analyzed using the SYNAPSE VINCENT volume analyzer (Fujifilm Medical Systems, Tokyo, Japan) (Figure 2). The residual FPFP area was semiautomatically selected and the volume was automatically calculated using this volume analyzer. Additionally, the residual ratio was calculated using the following formula: residual ratio $=$ residual FPFP volume $/$ resected FPFP volume. 

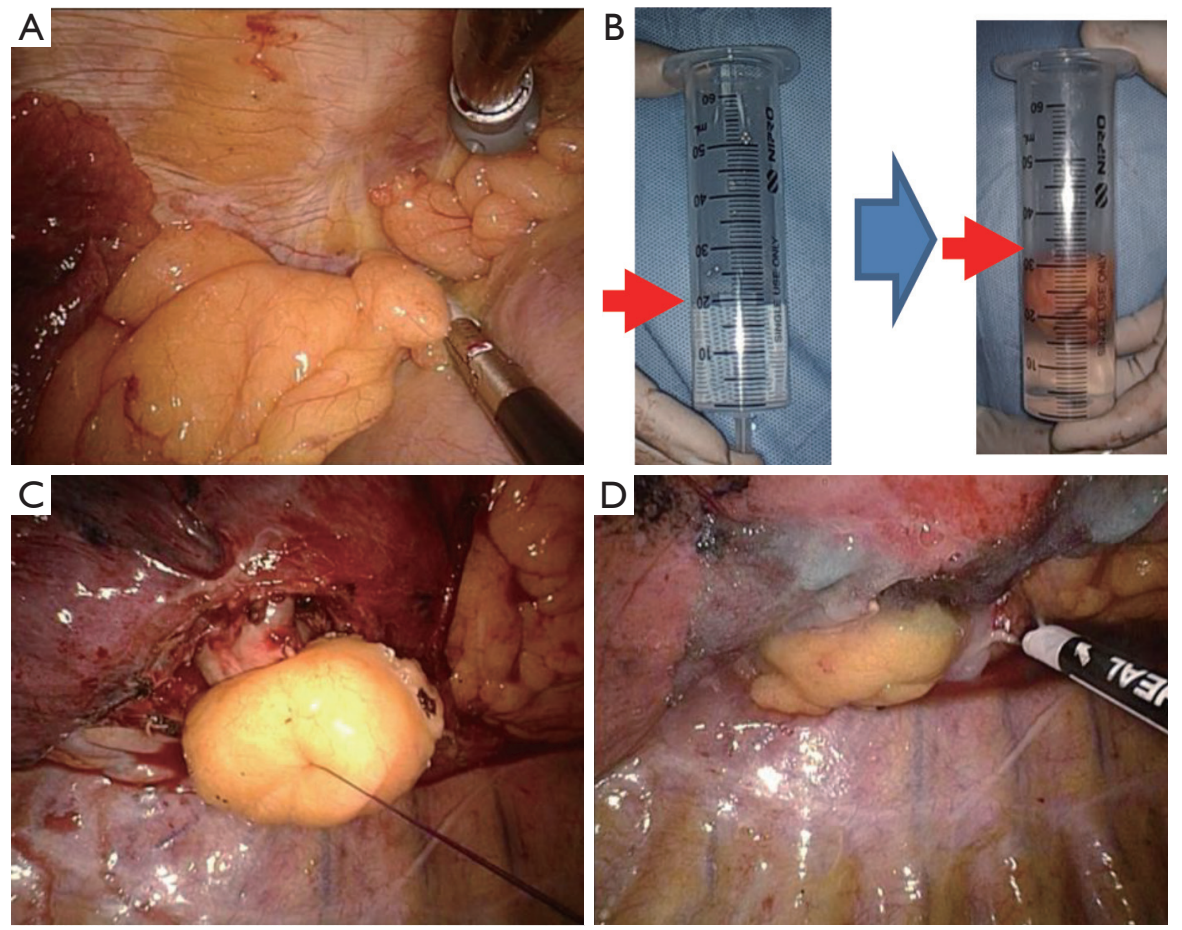

Figure 1 Management and FPFP measurement. (A) Thoracoscopic view of pericardial fat; (B) the resected fat was placed in a 50-mL syringe with $20 \mathrm{~mL}$ of saline to measure the volume; (C) the FPFP was stitched onto the bronchial stump using Prolene suture 3-0; (D) FPFP was fixed with fibrin glue and polyglycolic acid. FPFP, free pericardial fat pad.

\section{Statistical analyses}

Statistical analysis was performed using the SPSS Statistics version 23.0 software program (IBM Corporation, Armonk, NY, USA). Clinical variables are described as medians (ranges) for continuous variables and as frequencies (\%) for categorical variables.

An explorative analysis of the correlation between each preoperative clinical parameter, including surgical approach and resected FPFP volume, was performed using the MannWhitney $U$ test. Typically, the elderly population is defined as those aged 75 or older in terms of complications for lung cancer surgery; thus, the study population was divided into two groups with a cutoff of 75 years of age. We additionally predicted that lower body weight may affect the resected and remaining fat volume. However, although body mass index (BMI) values of less than $18.5 \mathrm{~kg} / \mathrm{m}^{2}$ are defined as indicating a low body weight by the World Health Organization, only two cases in the present study include BMI values of less than $18.5 \mathrm{~kg} / \mathrm{m}^{2}$ and this number was not enough to use for analysis. Instead, we used a BMI cutoff of $22 \mathrm{~kg} / \mathrm{m}^{2}$, which has been defined as a standard weight by the Japan Society for the Study of Obesity to stratify our population into two groups. Because N1 or higher is also a risk factor for BPF, we separated our population into two groups as $\mathrm{N} 0$ and $\mathrm{N} 1$ or $\mathrm{N} 2$ (1).

We used multiple regression analysis to confirm significant parameters, considering sex, BMI, and surgical approach as necessary variables to be entered into the model. Among our data, there was a significant correlation between the clinical $\mathrm{N}$ status and surgical approach $(\mathrm{P}<0.001$, chi-square test), with more cases with clinically advanced $\mathrm{N}$ status undergoing thoracotomy. Therefore, the clinical $\mathrm{N}$ status was excluded from the multivariate analysis. Multicollinearity was examined using variance inflation factors to evaluate the appropriateness of the models and parameter estimates. The normality of model residuals was checked through the visual inspection of probability-probability plots (data not shown).

The correlation between each clinical parameter, including postoperative factors and residual FPFP volume, was analyzed in the same manner. A Pearson correlation coefficient analysis was performed to detect correlations between two continuous parameters. 

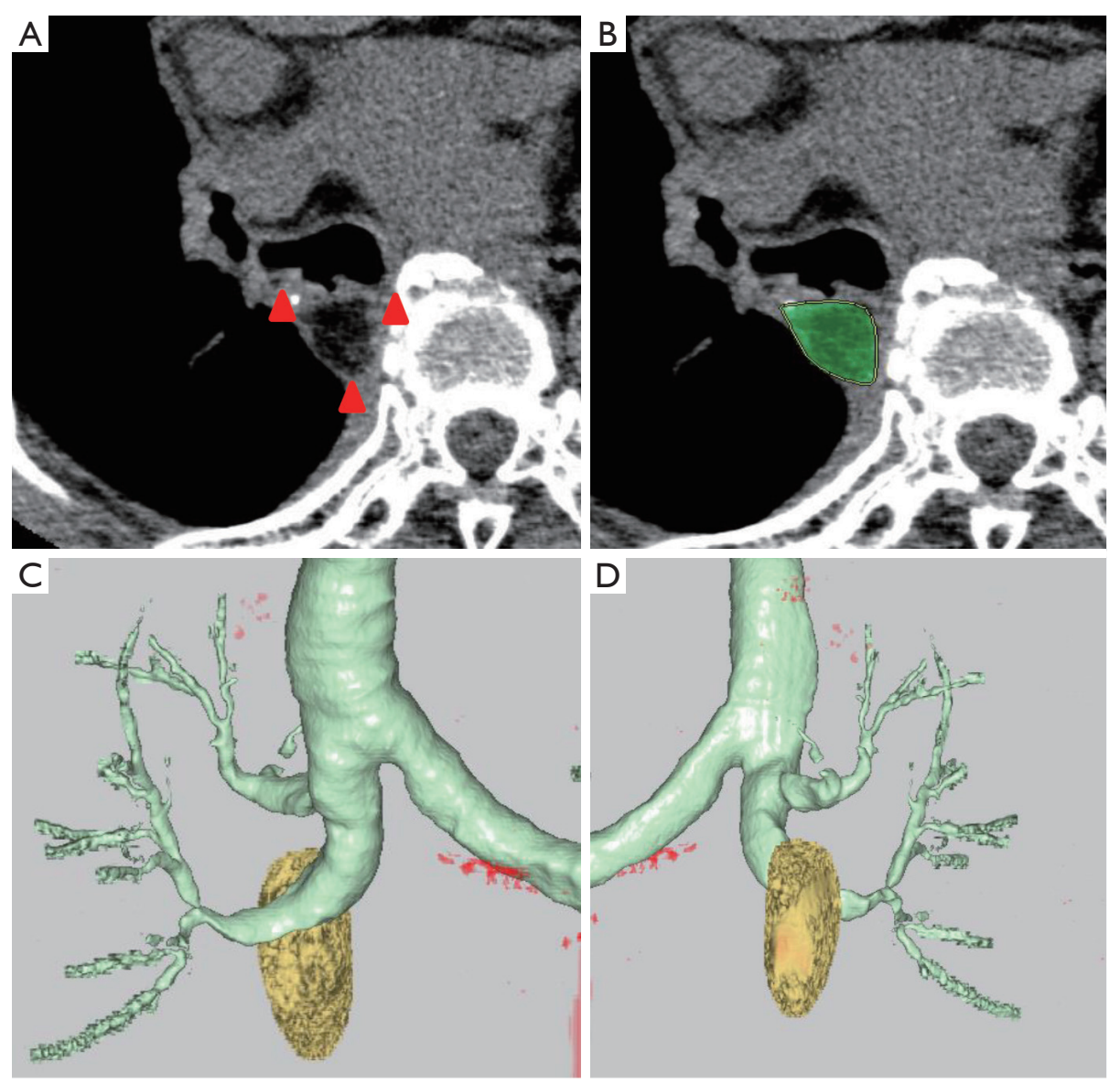

Figure 2 Chest CT and 3D-reconstructed images at 6 months after surgery. (A) A residual FPFP was observed as a low-density (red arrowheads) nodule around the bronchial stump; (B) the green area indicates the residual FPFP to be semiautomatically recognized using $3 \mathrm{D}$ imaging software; (C,D) the yellow nodule indicates the anterior (C) and posterior views (D) of the FPFP reconstructed using the 3D imaging software. CT, computed tomography; FPFP, free pericardial fat pad; 3D, three-dimensional.

\section{Results}

The median operation time for performing bronchial stump coverage with FPFP by VATS was 5 (3.5-8.0) min. Unfortunately, the procedure time was not measured for thoracotomy. There were no complications associated with FPFP harvest or stump coverage. Postoperative complications were observed in four cases (i.e., prolonged air leakage, chylothorax, atelectasis, and pneumonia in one case each). Neither BPF nor pyothorax occurred in this study.

According to our results, the median resected FPFP volume was $11[3-40] \mathrm{mL}$.

Table 2 shows the factors that may influence the volume of resected FPFP. In the univariate analysis, sex, BMI, surgical approach, resected lobe, lymph node dissection, clinical stage, and pathological stage were significantly associated with resected FPFP volume.

At this point, sex, BMI, and surgical approach were entered into the multivariate analysis, where it was found that BMI and surgical approach were significantly associated with the resected FPFP volume (Table 2).

Separately, the median residual FPFP volume was $4.3(0.4-15.5) \mathrm{mL}$. The median residual ratio was 0.39 (0.13-0.66) (Figure 3). The resected FPFP volume was significantly associated with the residual volume but not with the residual ratio $(\mathrm{P}<0.001$ and $\mathrm{P}=0.811$, Pearson correlation coefficient).

Further, there were no factors associated with the residual ratio (Table S1). Therefore, a multivariate analysis to elucidate significant parameters correlated with residual 
Table 2 Multivariate linear regression models for associations of clinical and surgical factors with harvested FPFP volume

\begin{tabular}{lccc}
\hline Variable & $\beta$ & $95 \% \mathrm{Cl}$ & $\mathrm{P}$ value \\
\hline Gender (men vs. women) & -2.431 & -6.623 to 1.762 & 0.247 \\
$\mathrm{BMI}\left(<21.9\right.$ vs. $\left.\geq 22.0 \mathrm{~kg} / \mathrm{m}^{2}\right)$ & 4.521 & $0.857-8.184$ & 0.017 \\
Surgical approach (VATS vs. thoracotomy) & 6.343 & $2.464-10.223$ & 0.002 \\
\hline
\end{tabular}

$\mathrm{R}^{2}=0.389$, VIF <1.16. FPFP, free pericardial fat pad; $\beta$, regression coefficient; Cl, confidence interval; BMI, body mass index; VATS, videoassisted thoracoscopic surgery; $\mathrm{R}^{2}$, coefficient of determination; VIF, variance inflation factor.

A

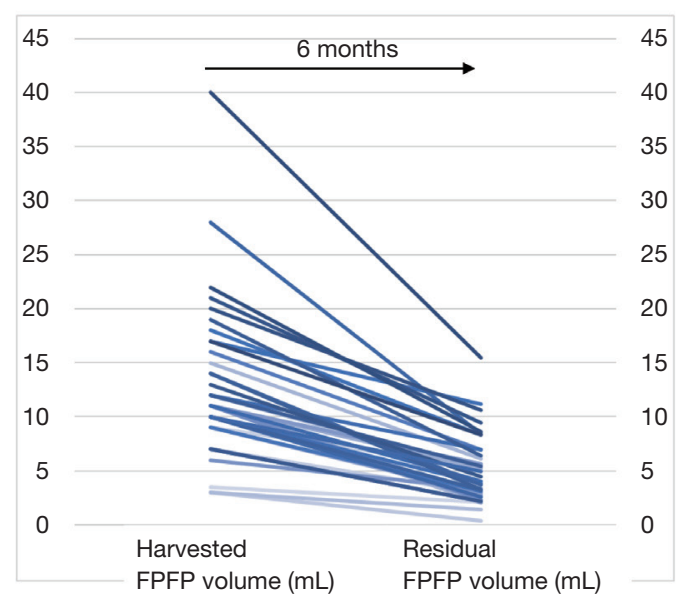

B

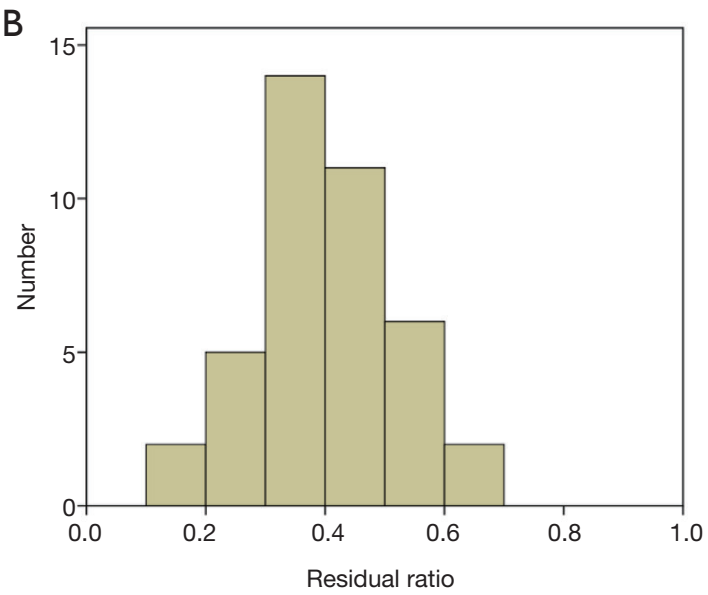

Figure 3 FPFP volume change and residual ratio. (A) Change in FPFP volume (mL) from resection to residual status at 6 months after surgery; (B) the number of cases according to the residual ratio. FPFP, free pericardial fat pad.

FPFP was not performed.

\section{Discussion}

In our study, using thin-slice CT and $3 \mathrm{D}$ reconstruction imaging, we confirmed that all FPFP grafts remained at 6 months after surgery but shrank to $40 \%$ within that time period. There were no clinical factors identified that influenced the residual FPFP ratio. Moreover, there were no complications associated with FPFP coverage or the occurrence of BPF in this study.

Of note, the resected FPFP volume in patients with high BMI values and those who underwent thoracotomy was markedly large among our study population. Generally, patients with high BMI values have more fat tissues, including pericardial tissue, and this may have contributed to this difference. For patients with low BMI values, we have two choices: one is to resect FPFP with thymic tissue, and the other is to select another covering material. Our results from this study are thus likely to be helpful in selecting the appropriate covering material before surgery. Thoracotomy was performed in more complex cases and patients at high risk for BPF onset. Moreover, the surgical view was magnified under a thoracoscope in these cases. These practices may have resulted in lower resected FPFP volumes among patients who underwent thoracotomy. Based on this result, a somewhat larger FPFP sample than is considered ideal should be resected during thoracoscopic procedures.

Regarding the residual FPFP ratio, our study showed that there were no clinical or surgical factors affecting the residual rate of FPFP. Paradoxically, since FPFP is expected to remain present to some extent in patients with any factors, it is likely that there will be no cases in which the FPFP will shrink excessively and become ineffective. A number of studies have confirmed the presence of FPFP after surgery through postoperative CT $(6,7,9)$. It was established that the volume of the FPFP gradually decreased with time. Matsuoka et al. demonstrated that residual FPFP in all cases can be observed through chest CT within 

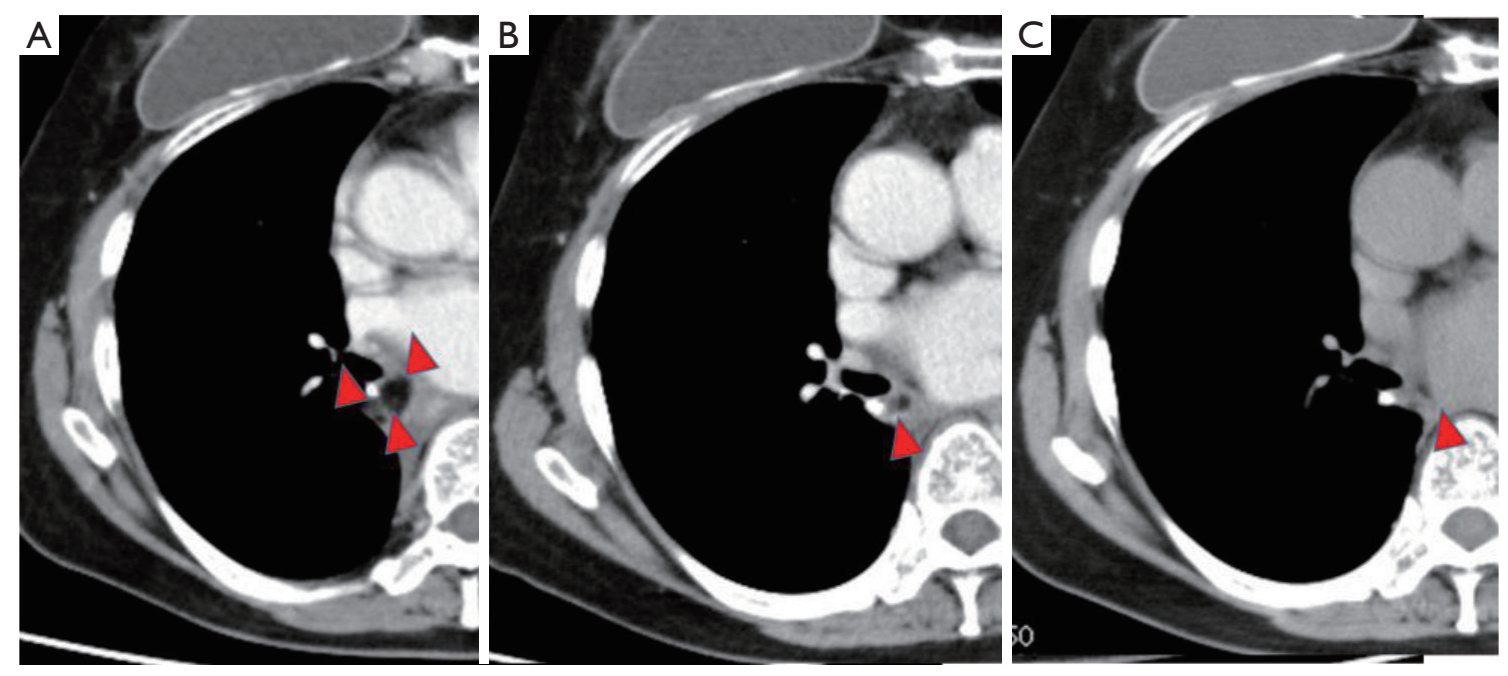

Figure 4 A long-term observation case. The presented CT images show the state of the residual FPFP at every 6 months until 18 months (red arrowheads). The harvested volume was $9 \mathrm{~mL}$ and the residual volumes at $6(\mathrm{~A}), 12(\mathrm{~B})$, and $18(\mathrm{C}) \mathrm{months}$ were $5.3 \mathrm{~mL}(58.6 \%), 3.0 \mathrm{~mL}$ (32.5\%), and $1.7 \mathrm{~mL}(19 \%)$, respectively.

5 months and 1 year after surgery in all and almost half of patients, respectively. In our study, the confirmation of the presence of residual FPFP was performed using thin-slice $\mathrm{CT}$ and $3 \mathrm{D}$ reconstruction imaging. Therefore, we were able to confirm its presence in all cases at 6 months. Since the occurrence of BPF at about 6 months after surgery is frequent (2), our data suggest the effectiveness of FPFP as a covering material of the bronchial stump during this period.

Thus far, only one study to our knowledge has investigated the residual ratio. Murakami et al. conducted a study using swine (10) wherein they harvested fat tissues and implanted them into the thoracic cavities of the animals. Six weeks after implantation, the fat tissues were resected. The investigators reported that no implanted fat tissues exhibited complications (e.g., abscess formation), and the residual ratio was approximately $70 \%$. In contrast, our study investigated humans rather than animals. Although it is almost impossible to measure the residual FPFP volume in humans in the same manner, the development of advanced 3D imaging made it possible to evaluate the intrathoracic tissue volume in this population noninvasively. In the present study, the residual ratio at 6 months after surgery was approximately $40 \%$. Among the study patients, one exemplar patient was checked using $3 \mathrm{D}-\mathrm{CT}$ every 6 months until 18 months, and this patient's residual volume was measured. In this individual, the harvested volume was found to be $9 \mathrm{~mL}$, while the residual volumes at 6,12 , and 18 months were 5.3 (58.6\%), 3.0 (32.5\%), and $1.7(19 \%) \mathrm{mL}$, respectively. It was confirmed that the FPFP gradually decreased over time (Figure 4). Therefore, the difference in the residual ratio is probably due to the variation in the observation period.

The most appropriate volume for resected FPFPs is currently unknown. In our study, $3 \mathrm{~mL}$ was the smallest volume of resected FPFP. Based on our experience, this volume appears to be the minimum required to ensure adequate coverage of the bronchial stump. Therefore, a residual FPFP volume of more than $3 \mathrm{~mL}$ may be desirable at 6 months after surgery to prevent the occurrence of BPF. Given that the resected FPFP shrank to $40 \%$ within 6 months in the present study, a harvested FPFP volume of $10 \mathrm{~mL}$ or greater may be ideal. Further, in patients with low BMI values and those undergoing VATS, it may be preferable to harvest larger amounts of pericardial fat, including the thymus.

The use of FPFP in this context is linked to some advantages. First, it is easy to handle even under thoracoscopy, as there is no need to consider the blood flow. Hence, the procedure requires approximately $5 \mathrm{~min}$ from harvest to coverage, and the use of FPFP exerted only a slight effect on the operation time. Second, this method is safely completed without any resultant functional defect.

A few studies to date have investigated angiogenesis related to FPFP and free fat application $(11,12)$. According to these studies, FPFP produces numerous kinds of angiogenic factors, and the intensity of neovascularization 
of pericardial fat was stronger than that observed for either omentum or muscle. Therefore, the investigators concluded that the use of FPFP is a reasonable option and may prevent the development of BPF. However, since all FPFP grafts shrank in our study, it is not clear as to whether angiogenesis is truly induced in humans. Meanwhile, the importance of adipose-derived stem cells (ASCs) has been emphasized for the reconstruction of breasts using a fat graft (13). ASCs are one of the components of adipose tissue, secreting angiogenic cytokines and accelerating wound healing (14).

FPFP can also be used as a surgical sealant in pulmonary resection. Ikeda et al. reported the efficacy of FPFP and the presence of fat at 6 months after surgery (9). This supports the tissue's repair ability and long-term effects of FPFP. Since FPFP is present for a long time regardless of blood flow, it may be effectively used in various situations.

A pedicle pericardial flap, including pericardiophrenic vessels, has been used in prior research to reinforce the bronchial stump (15). Even when using pericardial fat, the presence of the pedicle has been considered effective owing to the maintenance of the blood flow. However, Uramoto et al. demonstrated that the presence ratio of pericardial fat between pedicled and nonpedicled flaps was similar at approximately 6 months after surgery. Moreover, there were no cases of BPF reported in the two examined groups (6). According to this result, the use of FPFP with good operability may be more convenient. An intercostal muscle flap is an effective material in the coverage of the bronchial stump for the prevention of BPF. It is mainly used in patients undergoing thoracotomy. However, one study has reported its use in thoracoscopy (16). According to this study, the mobilization of the flap as overlying soft tissue may be challenging in obese patients, obscuring the surgical view. In contrast, the ease and extent of FPFP harvesting are greater in such cases. Moreover, in VATS approach cases, mobilization of the intercostal muscle flap is associated with damage to the thoracic wall and can impair respiratory function. It is because of the requirement of additional dissection of intercostal muscle that may have a respiratory function. Therefore, FPFP appears to be an easier and lessinvasive method, especially among patients undergoing thoracoscopy. The omentum is occasionally used for the prevention and treatment of BPF (17). However, while an effective material, its use involves an abdominal operation and so is considered the most invasive method. Thus, we only rely on the omentum as a source for tissue in the case of reoperation after lobectomy with BPF or right pneumectomy after chemoradiation.

\section{Limitations}

This study was a retrospective study. Measurement methods of FPFP were different between the intraoperative baseline and 6 months after surgery. This may result in some amount of error. To derive an accurate answer about the ideal FPFP size, it is necessary to show that there is a significant difference in the incidence of BPF between the two groups implanted with different FPFP sizes. However, since the incidence of BPF itself is low, the examination of many trials may be required. As an initial step, it seems to be meaningful to know the residual rate of FPFP. Our sample size was small, and we did not examine other materials including pedicled pericardial fat pad in this study; therefore, it was not possible to verify whether FPFP is the most effective covering material.

\section{Conclusions}

In all cases, residual FPFP was confirmed at 6 months after surgery and there were no cases of BPF reported in our study. Therefore, FPFP may constitute an appropriate material for covering the bronchial stump. The residual ratio was approximately $40 \%$ regardless of the patient's background. It is necessary to determine the volume of FPFP to be harvested, with careful consideration of the potential shrinkage that may occur within 6 months after surgery.

\section{Acknowledgments}

None.

\section{Footnote}

Conflicts of Interest: The authors have no conflicts of interest to declare.

Ethical Statement: The authors are accountable for all aspects of this work including ensuring that questions related to the accuracy or integrity of any part of the work are appropriately investigated and resolved. The institutional review board of Kanagawa Cancer Center approved this retrospective study (clinical registration no. 2019EKI-50). The requirement for patient consent was waived because of the retrospective nature of the study. 


\section{References}

1. Endo S, Ikeda N, Kondo T, et al. Risk assessments for broncho-pleural fistula and respiratory failure after lung cancer surgery by National Clinical Database Japan. Gen Thorac Cardiovasc Surg 2019;67:297-305.

2. Fuso L, Varone F, Nachira D, et al. Incidence and Management of Post-Lobectomy and Pneumonectomy Bronchopleural Fistula. Lung 2016;194:299-305.

3. Okuda M, Go T, Yokomise H. Risk factor of bronchopleural fistula after general thoracic surgery: review article. Gen Thorac Cardiovasc Surg 2017;65:679-85.

4. Di Maio M, Perrone F, Deschamps C, et al. A metaanalysis of the impact of bronchial stump coverage on the risk of bronchopleural fistula after pneumonectomy. Eur J Cardiothorac Surg 2015;48:196-200.

5. Getman V, Devyatko E, Abraham D, et al. Reconstitution of blood supply of the denuded bronchial stump. Ann Thorac Surg 2005;80:2063-9.

6. Uramoto H, Nakajima Y, Kinoshita H. Is the Isolated Pericardial Fat Pad Sufficient to Cover the Bronchial Stump and Separate the Pulmonary Artery in Order to Prevent Bronchopleural Fistula in Patients with Lung Cancer? Anticancer Res 2016;36:2385-9.

7. Matsuoka K, Imanishi N, Yamada T, et al. Clinical results of bronchial stump coverage using free pericardial fat pad. Interact Cardiovasc Thorac Surg 2016;23:553-9.

8. Takenaka T, Yamazaki K, Miura N, et al. The Prognostic Impact of Tumor Volume in Patients with Clinical Stage IA Non-Small Cell Lung Cancer. J Thorac Oncol 2016;11:1074-80.

9. Ikeda T, Sasaki M, Yamada N, et al. Controlling air

Cite this article as: Nagashima T, Ito H, Samejima J, Nemoto D, Eriguchi D, Nakayama H, Woo T, Masuda M. Postoperative changes of the free pericardial fat pad for bronchial stump coverage. J Thorac Dis 2019;11(12):5228-5236. doi: 10.21037/ jtd.2019.11.81 leaks using free pericardial fat pads as surgical sealant in pulmonary resection. Ann Thorac Surg 2015;99:1170-5.

10. Murakami J, Ueda K, Hayashi M, et al. The experimental validation of free fat grafts in thoracic surgery. Interact Cardiovasc Thorac Surg 2016;23:653-5.

11. Ichinose $\mathrm{Y}$, Asoh H, Yano T, et al. Use of a pericardial fat pad flap for preventing bronchopleural fistula: an experimental study focusing on the angiogenesis and cytokine production of the fat pad. Surg Today 1995;25:811-5.

12. Nishimura T, Hashimoto H, Nakanishi I, et al. Microvascular angiogenesis and apoptosis in the survival of free fat grafts. Laryngoscope 2000;110:1333-8.

13. Gentile P, Casella D, Palma E, et al. Engineered Fat Graft Enhanced with Adipose-Derived Stromal Vascular Fraction Cells for Regenerative Medicine: Clinical, Histological and Instrumental Evaluation in Breast Reconstruction. J Clin Med 2019. doi: 10.3390/jcm8040504.

14. Nie C, Yang D, Xu J, et al. Locally administered adiposederived stem cells accelerate wound healing through differentiation and vasculogenesis. Cell Transplant 2011;20:205-16.

15. Taghavi S, Marta GM, Lang G, et al. Bronchial stump coverage with a pedicled pericardial flap: an effective method for prevention of postpneumonectomy bronchopleural fistula. Ann Thorac Surg 2005;79:284-8.

16. Serna-Gallegos DR, McKenna RJ Jr. Video-Assisted Intercostal Muscle Flaps for Bronchial Stump Coverage. Ann Thorac Surg 2017;103:e215-7.

17. Jiang F, Huang J, You Q, et al. Surgical treatment for bronchopleural fistula with omentum covering after pulmonary resection for non-small cell lung cancer. Thorac Cancer 2013;4:249-53. 
Table S1 Correlation between clinicopathological factors, harvested FPFP volume, and FPFP residual ratio

\begin{tabular}{|c|c|c|c|c|c|}
\hline Clinicopathologic factors & Number & Median harvested volume (mL) & $P$ value & Median residual ratio & $P$ value \\
\hline Age (years) & & & 0.279 & & 0.469 \\
\hline$<74$ & 24 & 12.5 & & 0.41 & \\
\hline$\geq 75$ & 16 & 11.0 & & 0.36 & \\
\hline Gender & & & 0.004 & & 0.389 \\
\hline Male & 28 & 12.5 & & 0.40 & \\
\hline Female & 12 & 9.5 & & 0.34 & \\
\hline Smoking habit & & & 0.126 & & 0.753 \\
\hline Yes & 33 & 11.0 & & 0.39 & \\
\hline No & 7 & 9.5 & & 0.38 & \\
\hline $\operatorname{BMI}\left(\mathrm{kg} / \mathrm{m}^{2}\right)$ & & & 0.034 & & 0.498 \\
\hline$<21.9$ & 17 & 10.0 & & 0.35 & \\
\hline$\geq 22.0$ & 23 & 12.0 & & 0.43 & \\
\hline Diabetes mellitus & & & 0.781 & & 0.382 \\
\hline Yes & 7 & 11.0 & & 0.42 & \\
\hline No & 33 & 11.0 & & 0.37 & \\
\hline Hyperlipidemia & & & 0.570 & & 1.000 \\
\hline Yes & 12 & 13.0 & & 0.40 & \\
\hline No & 28 & 11.0 & & 0.38 & \\
\hline Cardiac diseases & & & 0.457 & & 0.209 \\
\hline Yes & 8 & 12.5 & & 0.33 & \\
\hline No & 32 & 11.0 & & 0.42 & \\
\hline Respiratory diseases & & & 0.396 & & 0.286 \\
\hline Yes & 18 & 11.0 & & 0.45 & \\
\hline No & 22 & 11.0 & & 0.37 & \\
\hline Clinical $\mathrm{N}$ status & & & 0.002 & & 0.512 \\
\hline 0 & 27 & 11.0 & & 0.42 & \\
\hline 1,2 & 13 & 17.0 & & 0.34 & \\
\hline Surgical approach & & & 0.003 & & 0.165 \\
\hline VATS & 23 & 11.0 & & 0.44 & \\
\hline Thoracotomy & 17 & 14.0 & & 0.34 & \\
\hline Resected lobe & & & - & & 0.138 \\
\hline Lower lobectomy & 30 & - & & 0.43 & \\
\hline Other & 10 & - & & 0.34 & \\
\hline Bronchoplasty & & & - & & 0.483 \\
\hline Yes & 9 & - & & 0.37 & \\
\hline No & 31 & - & & 0.42 & \\
\hline Lymph node dissection & & & - & & 0.345 \\
\hline LSD & 24 & - & & 0.44 & \\
\hline SND & 16 & - & & 0.36 & \\
\hline Postoperative complication & & & - & & 0.698 \\
\hline Yes & 6 & - & & 0.46 & \\
\hline No & 34 & - & & 0.39 & \\
\hline Pathological N status & & & - & & 0.864 \\
\hline 0 & 23 & - & & 0.41 & \\
\hline 1,2 & 17 & - & & 0.39 & \\
\hline Postoperative pleurodesis & & & - & & 0.450 \\
\hline Yes & 1 & - & & 0.31 & \\
\hline No & 39 & - & & 0.39 & \\
\hline
\end{tabular}

FPFP, free pericardial fat pad; BMI, body mass index; VATS, video-assisted thoracoscopic surgery; LSD, lobe-specific nodal dissection; SND, systemic nodal dissection. 